\title{
Extraction Optimization for Phenols and Flavonoids from Cultured Mycelia of Cordyceps Ophioglossoides and Exploration of Bioactivities of its Aqueous and Ethanol Extracts
}

\author{
Qinqin Xu' ${ }^{1,2}$, Zhao Zhao ${ }^{1,3}$, Yisheng Sun ${ }^{1,4}$, Ryan P Mackay ${ }^{2}$ and Yong-Quan Li*1 \\ ${ }^{1}$ Institute of Pharmaceutical Biotechnology\& First Affiliated Hospital, Zhejiang University School of Medicine, Hangzhou 310058, China
}

${ }^{2}$ Departments of Otolaryngology, Molecular and Cellular Physiology, Feist-Weiller Cancer Center, LSU Health Shreveport, 1501 Kings Highway, Shreveport, LA 71103, USA

${ }^{3}$ Centre of Laboratory Medicine, Zhejiang Provincial People's Hospital,People's Hospital of Hangzhou Medical Collage, Hangzhou, Zhejiang, 310014, China ${ }^{4}$ Zhejiang Provincial Center for Disease Control and Prevention, 630 Xincheng Road, Hangzhou, Zhejiang, 310051, China

Received: 制: November 20, 2018; Published: 制: November 30, 2018

*Corresponding author: Yong Quan Li, Institute of Pharmaceutical Biotechnology \& First Affiliated Hospital, Hangzhou 310058, China

\begin{abstract}
Aqueous and ethanol extracts from the mycelia of Cordyceps Ophioglossoides have been used as a nutritional supplement, especially for women suffering from massive postpartum vaginal bleeding in Southwest China. However, there is limited information regarding its components, extraction conditions and bioactivities. The present study was undertaken to identify total phenol and flavonoid content of cultured mycelia of Cordyceps Ophioglossoides under optimized extraction condition. The anti-oxidative activities of two extracts were evaluated in three in vitro systems including 1, 1-diphenyl-2-picrylhydracyl (DPPH) radical scavenging assay, superoxide radical scavenging assay, and hydroxyl radical scavenging assay. The estrogenic activity of the $20 \%$ ethanol extracts $(20 \% \mathrm{EE})$ was studied by cell proliferation assay, by luciferase reporter assay and by measuring expression of ER endogenous target genes in human breast cancer cells. These results suggest that the extracts of Cordyceps Ophioglossoides have high levels of phenols and flavonoids as well as anti-oxidative and estrogenic bioactivities.
\end{abstract}

Keywords: Cordyceps Ophioglossoides; Phenol; Flavonoid; Aqueous and Ethanol Extracts; Anti-Oxidative Activity; Estrogenic Activity

Abbreviations: EE: Ethanol Extracts; SERM: Selective Estrogen Receptor Modulators; DPPH: Diphenyl-2-Picrylhydracyl; TBHQ: Tert-Butylhydroquinone; BHT: Butyl-4-Methylphenol; E2: Estradiol; TPC: Total Phenols Content; TFC: Total Flavonoid Content; AE: Aqueous Extracts MTT, 3-(4,5-Dimethylthiazol-2-yl)-2,5-Diphenyl Tetrazolium Bromide; ROS: Reactive Oxygen Species

\section{Introduction}

Cordyceps are commonly known in China as a medical and nutritional supplement. Cordyceps Ophioglossoides is a unique species of Cordyceps which primarily parasitizes certain types of Elaphomyces. Cordyceps Ophioglossoides has been used as fungal medication in gynecology, mostly for women with massive postpartum vaginal bleeding in ancient China [1,2]. However, the main functional components and bioactivities were largely unknown due to the rarity of wild Cordyceps Ophioglossoides. It is not only small in size but also grows extremely slowly in a particular climate and host. The low yield of natural product has made it hard for researchers to study its activity. In our previous studies, we developed a large-scale submerged culture method for Cordyceps
Ophioglossoides and obtained a large number of the mycelia which made it convenient for us to analyze the composition and activity of mycelia extract $[3,4]$. The extract of Cordyceps has been studied for its high capacity in scavenging free radicals which are implicated in various diseases [5-7].

It has been revealed that the extract is rich in phenol and flavonoid compounds and has a high antioxidant capacity, which is known to play important roles in the human metabolic system and protect against cardiovascular and neurodegenerative disease [8-10]. Phenols and flavonoids are considered phytoestrogens, a class of naturally produced chemicals that act as selective estrogen receptor modulators (SERMs). SERMs are used by women who 
suffer climacteric and menopausal syndrome as estrogen therapy can alleviate these symptoms. However, phytoestrogens lead to an undesirable increase in risk of cancer [11-15]. Studies on Cordyceps Ophioglossoides extract are lacking, and its activities are not clear. In this study, we optimized the extraction conditions for total phenols and flavonoids from cultured mycelia of Cordyceps Ophioglossoides. We then studied the anti-oxidative activities of the extracts by using 1,1-diphenyl-2-picrylhydracyl (DPPH) radical scavenging assay, superoxide radical scavenging assay and hydroxyl radical scavenging assay. Finally, the estrogen-like activity of the cultured Cordyceps Ophioglossoides extracts was determined by proliferation assay of ER +/- cells and expression of ER target genes.

\section{Materials and Methods}

\section{Chemical}

Tert-butylhydroquinone (TBHQ, purity $>98 \%$, Fluka AG), 2, 6-di-tert-butyl-4-methylphenol (BHT, purity >99\%), rutin, 3-(4,5-Dimethylthiazol-2-yl)-2,5-diphenyl tetrazolium bromide (MTT), 17 $\beta$-estradiol (E2) ,1,1-diphenyl-2-picrylhydrazyl (DPPH), and Folin-Ciocalteu's phenol were purchased from Sigma-Aldrich (St. Louis, MO, USA). Gallic acid monohydrate was obtained from Sinopharm Chemical Reagent Co., Ltd. (Shanghai, China). All solvents and other reagents were of analytic grade.

\section{Mycelia Cultivation}

The mycelia of Cordyceps Ophioglossoides were obtained from large scale fermentation in the medium as optimized by our lab [4]. Each shaking-flask culture was carried out in 1L Erlenmeyer flasks containing $200 \mathrm{~mL}$ of medium and incubated at $23-25^{\circ} \mathrm{C}$ for 7 days. Thereafter, 1-1.5 L of shaking-flask cultures was inoculated into a 3-ton fermentation tank containing 1 ton of cultural media. Cordyceps Ophioglosoides was cultured at $23-25{ }^{\circ} \mathrm{C}$ for 2 days. Finally, it was inoculated into a 20-ton fermentation tank containing 12 tons of cultural media. After 3 days of growth, the mycelia were fileted and lyophilized to a powder and stored at $-20{ }^{\circ} \mathrm{C}$ for use.

\section{Determination of Total Phenols Content (TPC) and Total Flavonoid Content (TFC)}

Total phenol content of the extract was quantified using FolinCiocalteau's reagent according to the method reported by Mau et al. [16] with some modifications. Briefly, $2 \mathrm{~mL}$ of extract and deionized water mixture were added to $4 \mathrm{~mL}$ of $2 \%$ aqueous sodium carbonate solution. After 3 minutes, $100 \mu \mathrm{l}$ of Folin-Ciocalteau reagent was added to the mixture. After 30 minutes, absorbance was measured at $750 \mathrm{~nm}$ against a blank. The content of total phenols was calculated on the basis of the calibration curve of gallic acid. The total flavonoid content of extracts was determined according to the method reported by Jia et al. [17] with slight modifications. In short, $5 \mathrm{~mL}$ of extract and deionized water mixture were placed in a 10 $\mathrm{mL}$ volumetric flask, followed by addition of $0.3 \mathrm{~mL}$ of $5 \%$ sodium nitrite. After 6 minutes, $0.3 \mathrm{~mL}$ of $10 \%$ aluminum chloride was added. After 6 minutes, $4 \mathrm{~mL}$ of $1 \mathrm{M}$ sodium hydroxide was added, and the solution was diluted to $10 \mathrm{~mL}$ with deionized water. After 15 minutes, the solution was measured at $505 \mathrm{~nm}$. The flavonoid content was calculated on the basis of the calibration curve of rutin.
To optimize the extraction methods for the phenol and flavonoid, we used the one-factor-at-a-time method.

\section{Anti-0xidative Activities Measurement}

1-Diphenyl-2-Picrylhydracyl Radical Scavenging Assay: The free radical scavenging activity of the extracts toward 1, 1-diphenyl-2-picryl-hydrazil (DPPH) was examined according to the method reported by Shimada et al. [18]. A $0.2 \mathrm{mmol} / \mathrm{l}$ solution of DPPH radical in ethanol was prepared and $2 \mathrm{~mL}$ of this solution was added to $2 \mathrm{~mL}$ of water solution containing different volumes of $100 \mathrm{mg} / \mathrm{mL}$ aqueous extracts (AE) or aqueous and ethanol extracts $(20 \% \mathrm{EE})(25,50,100,150,200 \mu \mathrm{l})$. After 30 minutes, the absorbance was measured at $517 \mathrm{~nm}$. Low absorbance of the reaction mixture indicated higher free radical scavenging activity. The scavenging of DPPH radical in percentage was calculated by the following equation: Scavenging activity $(\%)=(1-\mathrm{A} 1 / \mathrm{A} 0) \%$, where $\mathrm{A} 0$ was the absorbance of the control reaction and $\mathrm{A} 1$ was the absorbance in the presence of extracts $(25,50,100,150,200$ $\mu \mathrm{l}) .1 \mathrm{~g} / \mathrm{L} \mathrm{BHT}$ and $1 \mathrm{~g} / \mathrm{L}$ TBHQ were used as positive controls.

Superoxide Radical Scavenging Assay: Scavenging of superoxide by antioxidants was estimated by the inhibition of pyrogallol autoxidation as described previously [19] with some modifications. In brief, $5 \mathrm{~mL}$ of $50 \mathrm{mmol} / \mathrm{L}$ Tris-HCl buffer (pH 8.2) and $3.8 \mathrm{~mL}$ of distilled water and $100 \mathrm{mg} / \mathrm{mL} \mathrm{AE}$ or $20 \%$ EE extracts $(50,100,150,200 \mu \mathrm{l})$ were incubated at $25{ }^{\circ} \mathrm{C}$ for 10 minutes and followed by an addition of $200 \mu \mathrm{l}$ of pyrogallol at the same temperature for 5 minutes. The absorbance of the mixture at 322 $\mathrm{nm}$ per $30 \mathrm{~s}$ for 4 minutes against the blank and the autoxidation speed was calculated as absorbance/minute. The percent inhibition of superoxide anion radicals scavenging was calculated using the following formula: Inhibition pyrogallol autoxidation $(\%)=[1-\mathrm{A} 1 /$ $\mathrm{A} 0] \times 100$, where A0 is the autoxidation speed of control without the tested samples and $\mathrm{A} 1$ is the autoxidation speed in the presence of the tested samples. BHT and $0.2 \mathrm{mg} / \mathrm{mL}$ ascorbic acid were used as positive controls.

Hydroxyl Radical Scavenging Activity: Hydroxyl radical scavenging activity was measured according to the method described by [20] with some modifications. The reaction mixture containing $0.2 \mathrm{mM}$ EDTA-Fe (3.0 mL), $3.0 \mathrm{mM} \mathrm{H} 2 \mathrm{O} 2(3.0 \mathrm{~mL})$ and $2.0 \mathrm{mM}$ salicylic acid ( $3 \mathrm{~mL}$ ) was incubated at $37^{\circ} \mathrm{C}$ water bath for 15 minutes. It was then added to $1 \mathrm{~mL}$ distilled water and sample mixture containing $100 \mathrm{mg} / \mathrm{mL}$ extracts $(50,100,150,200,250$ $\mu \mathrm{l}$ ) for 60 minutes at $37{ }^{\circ} \mathrm{C}$. Hydroxyl radicals were detected by monitoring absorbance at $510 \mathrm{~nm}$. In the control, sample was substituted with distilled water and sodium phosphate buffer instead of H2O2. Results were calculated with the following equation: Scavenging effect $(\%)=[1-\mathrm{A} 1 / \mathrm{A} 0] \times 100$, where $\mathrm{A} 0$ was the absorbance of the control reaction and $\mathrm{A} 1$ was the absorbance in the presence of extracts $(50,100,150,200,250 \mu \mathrm{l})$.

\section{Cell Culture Conditions}

MCF-7 and MDA-MB-231 cells were maintained in Dulbecco's Modified Eagle medium (DMEM)/high glucose media with $10 \%$ heat-inactivated FBS, sodium pyruvate (1\%), penicillin- 
streptomycin (1\%), and glutamax-1 (1\%). Three days prior to treating the cells with the extracts or estrogen, the medium was replaced with phenol red-free, DMEM/ high glucose medium containing charcoal/dextran-stripped serum.

\section{Cell Proliferation Assay}

Cell proliferation was determined using a standard colorimetric MTT (3-4, 5-dimethylthiazol-2-yl-2, 5-diphenyl-tetrazolium bromide) reduction assay [21]. MCF-7 and MDA-MB-231 cells were cultured in phenol red-free medium containing $10 \%$ charcoal dextran-treated FBS. $8 \times 103$ cells were spread onto 96-well culture plates and were incubated for 3 days with $17 \beta$-estradiol $(5 \times 10$ ${ }^{8} \mathrm{M}$ ) and $20 \% \mathrm{EE}(50,100,200,400 \mu \mathrm{g} / \mathrm{mL})$. Cell viability was measured in terms of the absorbance at $490 \mathrm{~nm}$ with a microplate reader (Synergy Mx Monochromator-Based Multi-Mode Microplate Reader, Winooski, VT). All experiments were performed in triplicate. For each treatment, cell viability was evaluated as a percentage using the following equation: cell proliferation rate (\%) =OD of the experimental samples/OD of the control $\times 100$.

\section{Luciferase Assay}

MCF-7 cells were first grown in phenol red-free medium containing $10 \%$ charcoal dextran-treated FBS and plated in 24-well plates $(1.5 \times 105 /$ well). pERE-TK-Luc or control pTK-luc plasmids were cotransfected with pRLuc plasmids, which served as the internal control. Transfections were conducted using oligofectamine 2000 (Invitrogen, Carlsbad, CA) following the manufacturer's instructions. Four hours post transfection, $17 \beta$-estradiol $\left(5 \times 10^{-8}\right.$ M), $20 \% \mathrm{EE}$, or an equal amount of vehicle were added. Luciferase assays were carried out using a Dual-Luciferase Assay Kit (Promega, Madison, WI) after additional 36 hours.

\section{Real-Time PCR}

Cells were plated in $3.5-\mathrm{cm}$ plastic dishes $\left(2 \times 10^{5}\right.$ cells/ dish in $2 \mathrm{~mL}$ of estrogen-free medium) for 24 hours and treated with the test compounds (17 $\beta$-estradiol, 20\% EE) for 1 hour. Total RNA was isolated by a RNeasy Mini Kit (QIAGEN). 500 ng RNA of each sample was reverse transcribed to cDNAs for real-time PCR analyses. The expression of representative ER $\alpha$ target genes (pS2, cyclin D) were assessed using the following primers: 5'- TCCCAGTGTGCAAATAAG $-3^{\prime}$ (pS2-F) and 5'ATTCACACTCCTCTTCTGG-3' (pS2-R), 5'- CGCCCCACCCCTCCAG-3' (Cyclin D-F) and 5'- CCGCCCAGACCCTCAGACT-3' (Cyclin D-R). 18s rRNA expression levels were also measured simultaneously for normalization. The results were expressed as fold inductions.

\section{Statistical Analysis}

Every experiment was repeated in triplicate, and the results were presented as mean values \pm SD (standard deviations). Differences at $\mathrm{p}<0.05$ were considered to be significant.

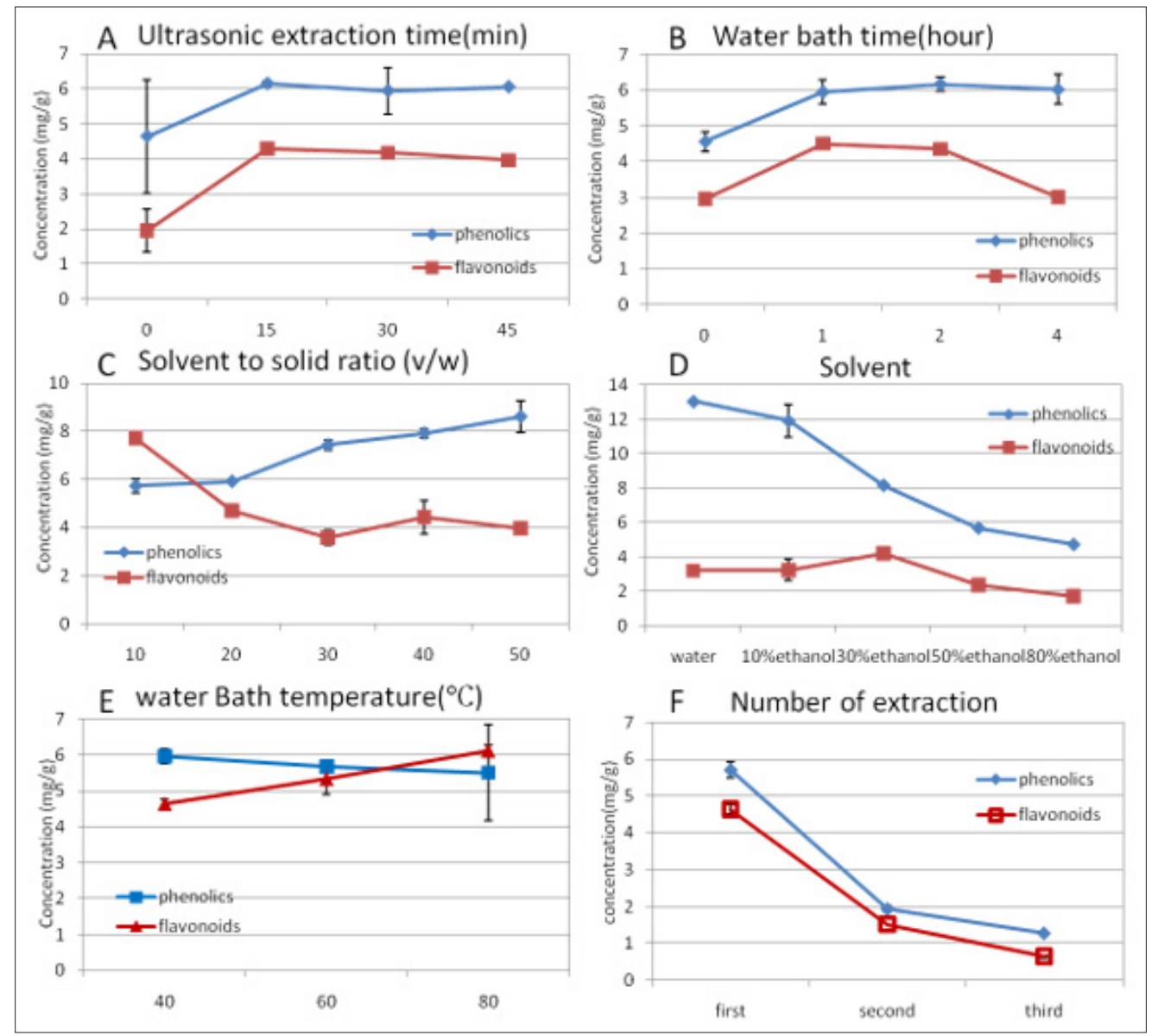

Figure 1: Influence of extraction conditions on phenol and flavonoid concentrations from mycelia. Values are the mean of three separate experiments. 


\section{Results and Discussion}

\section{Effect of the Extraction Conditions on Total Phenol Content (TPC) and Total Flavonoid Content (TFC)}

Antioxidant activities of phenols and flavonoids have been recognized for decades, and it appears that the content of these two compounds are important for the quality of Cordyceps. The effect of extraction conditions on phenol and flavonoid concentration from the mycelia of Cordyceps Ophoglossoides were previously unknown, and we have optimized the extraction method for the first time. Figure 1. showed the influence of extraction time on flavonoid and phenol content was similar. There was no significant difference in extraction between ultrasonic time, but it was much higher than no ultrasonic application. One hour in $80^{\circ} \mathrm{C}$ was selected as optimum time for phenol and flavonoid extraction from mycelia which is consistent to the research by Al-Farsis and Lee (2008). The TPC and TFC extracted by five different solvents to solid ratio $(\mathrm{v} / \mathrm{w})$ $(10: 1,20: 1,30: 1,40: 1$ and 50:1) is shown in Figure 1C. The result of phenol extraction was the same as previous studies from date seeds by Al-Farsis et al. while the flavonoid extract had the opposite result. Higher solvent volume was better for yield of TPC; however, it was neither convenient nor economical. Therefore, the 10:1 ratio and two times extraction were selected for the optimal solvent to solid ratio. Figure 1D. shows that high ethanol ratio was unfavorable for extraction of these two compounds.

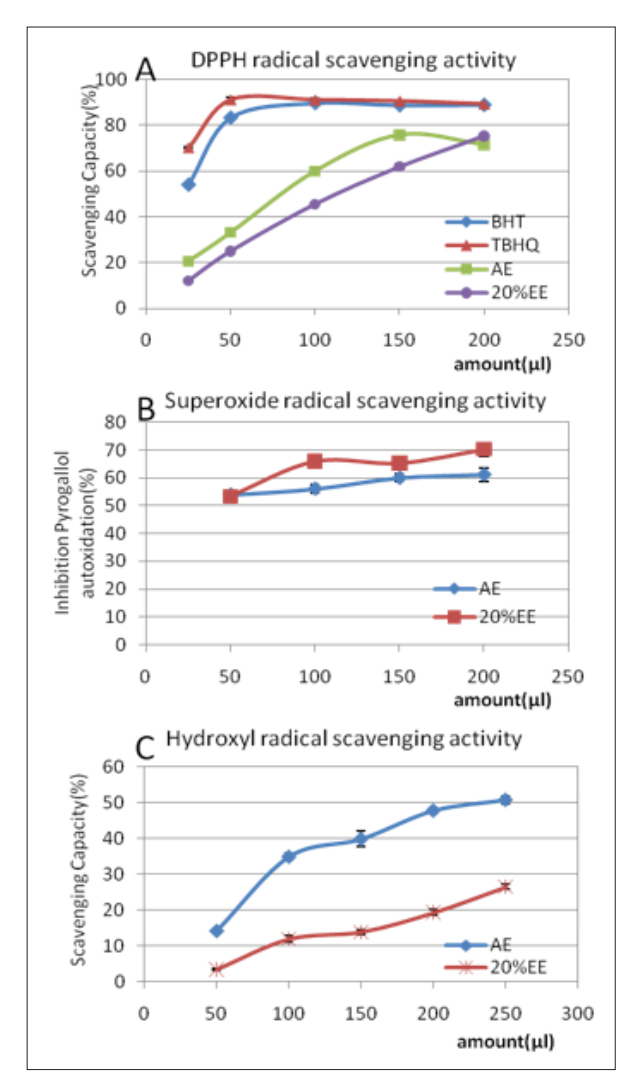

Figure 2: Scavenging effect of $\mathrm{AE}$ and $20 \% \mathrm{EE}$ on $\mathrm{DPPH}$, superoxide radical and hydroxyl radical. Each value is the mean \pm SD of triplicate measurements. (A) DPPH radical scavenging activity; (B) superoxide radical scavenging activity; (C) Hydroxyl radical scavenging activity.
It is consistent with that of Matthaüs (2002) who found that extraction with water gave the highest amounts of phenol compounds from fat-free residues of different oil seeds. The highest flavonoid content was obtained at 30\% ethanol while the highest content of phenol was obtained at aqueous extraction. Finally, 20\% EE extraction was picked for estrogenic activity assay for a balance between phenol and flavonoid concentration. Temperatures from 40 to $65^{\circ} \mathrm{C}$ were selected as the optimum temperatures to extract phenols from various plants. Farsis et al. [22] also reported that heat enhanced the recovery of phenol compounds. In Figure 2 , it appears that increasing the temperature from 40 to $80{ }^{\circ} \mathrm{C}$ correlates with low phenol content and high flavonoid content. The phenols might be denatured in the high temperature. The effect of temperature on flavonoid concentration was in agreement with the theory that increasing temperature favored extraction by enhancing both the solubility of solute and the diffusion coefficient. For the total content of the two compounds, $80{ }^{\circ} \mathrm{C}$ was considered to be optimal. The selected optimum condition for extracting phenols and flavonoids from mycelia was $20 \%$ EE to solid ratio (v/w) 10, ultrasonic extraction 20 minutes, kept in $80^{\circ} \mathrm{C}$ for 1 hour, two times extraction. Under the optimized condition, there were $21.8 \mathrm{mg}$ TP and $13.2 \mathrm{mg}$ TF per gram of mycelia (equal to $87.3 \mathrm{mg}$ TP and 53.04 mg TF per gram of extraction).

\section{Anti-Oxidative Activities of the Extracts from Cordyceps Ophioglossoides}

The DPPH radical scavenging model is a widely used method to evaluate natural antioxidant activities in a relatively short time. The DPPH radical scavenging activity of the extract was evident in all tested concentrations but lower than that of TBHQ and BHT (Figure 2A). It was observed that the scavenging effect of $\mathrm{AE}$ increased linearly with increasing concentration. The percentage of DPPH radical scavenging activity at $200 \mu \mathrm{l}$ volume of $20 \% \mathrm{EE}$ was $75.4 \%$. AE showed the best scavenging activity with $75.7 \%$ at $150 \mathrm{ul}(100 \mathrm{mg} /$ $\mathrm{mL}$ ) added. Among reactive oxygen species (ROS), superoxide is a relatively weak oxidant. However, it may decompose to form stronger ROS such as hydroxyl radical, which may participate in lipid peroxidation and damage cellular membranes. Therefore, superoxide scavenging is extremely important to antioxidant function. To quantify the extent of superoxide scavenging activity, we determined the inhibition of pyrogallol autoxidation. Changes in color represent the content of superoxide radicals and indicate the antioxidant activity of the sample. As Figure 2B. shows, 50\% to $70 \%$ inhibition of pyrogallol autoxidation was detected.

There were no direct relationships between concentration and activity. This inhibitory rate was similar to that of ascorbic acid (63.2\%) and stronger than BHT (25.2\%). AE and 20\% EE both exhibited scavenging activity of hydroxyl radical in a concentrationdependent manner as shown in Figure 2C. Scavenging ratios improved with increasing extract concentration. The highest value (50.7\% in AE, $26.5 \%$ in $20 \%$ EE, respectively) was lower than ascorbic acid (70\%). The scavenging effects of AE on hydroxyl radical were much higher than $20 \% \mathrm{EE}$. It is interesting that BHT showed a lower activity in autoxidation which is consistent to the study by Kim et. al [23]. BHT in various solvents showed significant 
differences in scavenging activity [24]. The extracts from cultured mycelia have direct and potent antioxidant activities. There may be some interactions and synergistic effects of the extracts rich in polysaccharide, phenol and flavonoid compounds. Any of them may be the reason for the antioxidant properties. Total antioxidant power is more meaningful to evaluate health benefits because of the cooperative action of antioxidants as reported by Celik et al. [24]. Furthermore, the use of natural extracts is more economical than the use of synthetic derivatives and reduces environmental pollution.
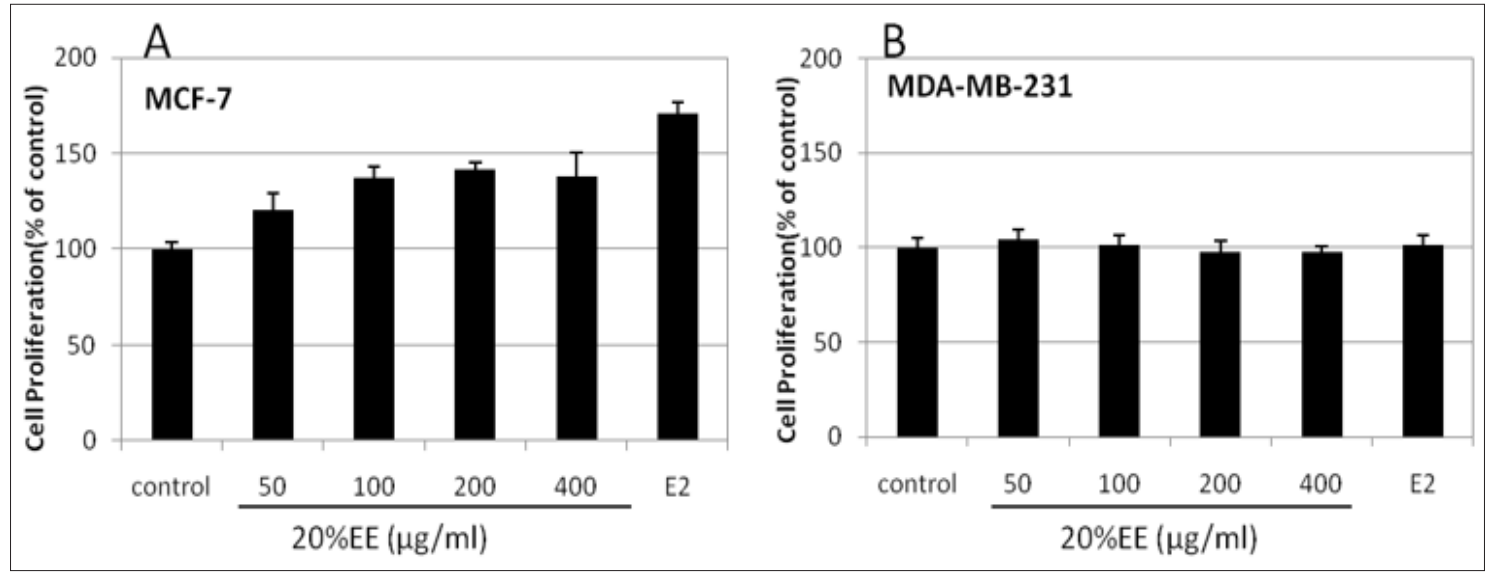

Figure 3: Estrogenic activity of 20\%EE on the rate of MCF-7 (A) and MDA-MB-231 (B) cell proliferation. The MTT assay was determined at 72 hours after treatment. Each point is the mean \pm S.D. of triplicate measurement. Values marked by *; **, are significantly different $(\mathrm{p}<0.05 ; \mathrm{p}<0.01)$.

\section{Estrogenic Activity of the Extracts from Cultured Mycelia of Cordyceps Ophioglossoides}

Effects of $20 \%$ EE on the Growth of ER (+/-) Human Breast Cancer Cells: The aqueous and ethanol extracts of Cordyceps Ophioglossoides have been widely used in southwest China for women with irregular menstruation which lead us to study the estrogenic activity of the mycelia. Estrogenic activity of $20 \% \mathrm{EE}$ was analyzed by measuring the rate of MCF-7 cell proliferation in response to various concentrations of the extracts $(50,100,200$, $400 \mu \mathrm{g} / \mathrm{mL}$ ). As shown in Figure 3A, extracts produce a time and dose response in the proliferation of MCF-7 cells. However, the extracts did not affect the growth of MDA-MB-231 cells which is an ER-negative cell line, as shown in Figure 3B. This suggests that the extracts may stimulate the proliferation of MCF-7 through an ER- dependent mechanism.

Effect of 20\% EE on the Activation of ER: The effect of $20 \%$ EE on the activation of ER was further studied using a luciferase reporter gene assay. ER-positive MCF-7 cells were transiently transfected with ERE-TK-Luc and control TK-Luc, while pRLuc was also included as a control for transfection. As shown in Figure 4, treatment with $20 \%$ EE extract ranged from 50 to $400 \mu \mathrm{g} / \mathrm{mL}$ and induced a significant increase in ERE-luciferase reporter activity compared with control. The effect was also dose-dependent with a maximal activity at $100 \mu \mathrm{g} / \mathrm{mL}$. These results confirmed our hypothesis that the extracts of mycelia from cultured Cordyceps ophioglossoids have estrogenic activity through a classical estrogen response pathway by interaction with ER.
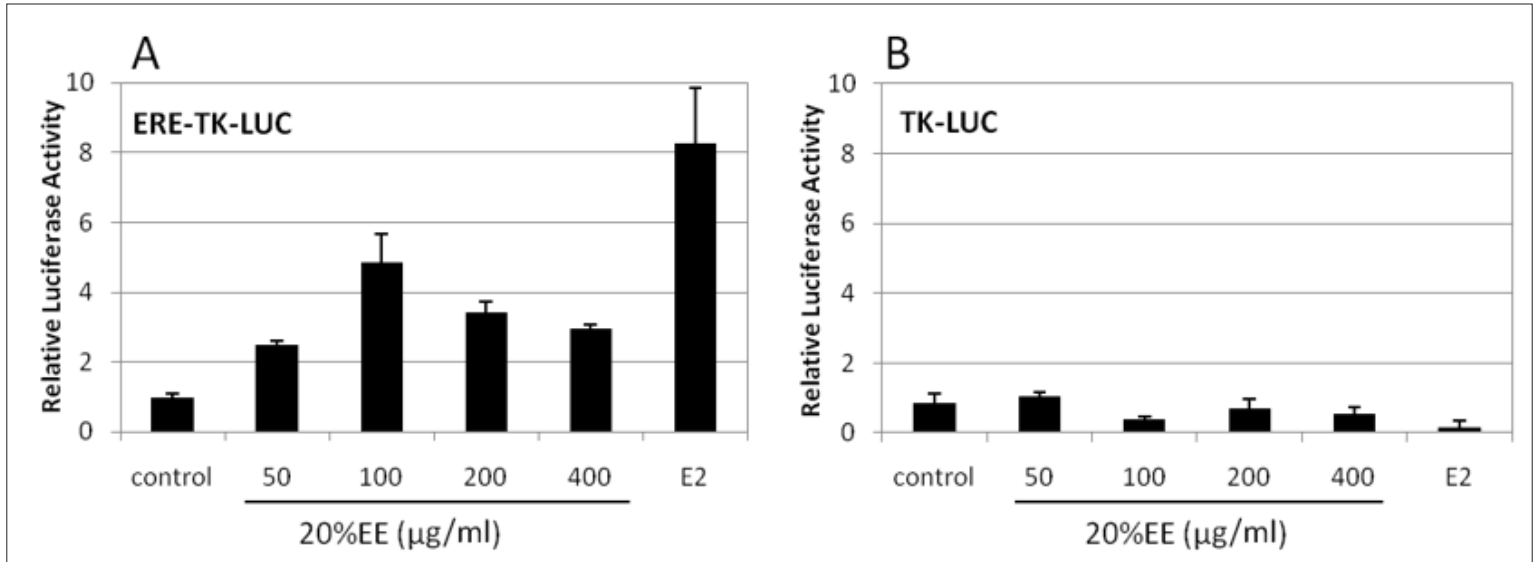

Figure 4: Luciferase assay for confirming the estrogen like activity of 20\%EE. MCF-7 cells were transfected with ERE-TK-LUC (A) and TK-LUC (control) (B) in the presence of $20 \% \mathrm{EE}$, and estrogen was used as a positive control. All luciferase activities were an average value \pm standard deviation $(\mathrm{SD})$. Values marked by *; **, are significantly different $(p<0.05 ; \mathrm{p}<0.01)$. 
Effects of 20\% EE on the mRNA Levels of PS2 and Cyclin D in MCF-7 Cells: ER regulates gene expression through interaction with DNA sequences termed estrogen response elements located within the regulatory regions of target genes such as pS2 and cyclin D. To further confirm that the extract exhibits estrogenic activity, we evaluated the expression of well-known ER endogenous target genes pS2 and cyclin D. Our results indicated that the extract significantly up-regulates mRNA expression of both pS2 (Figure 5A) and cyclin D (Figure 5B). These results are consistent with above MTT and luciferase assays and support the estrogenic activities of the extract.
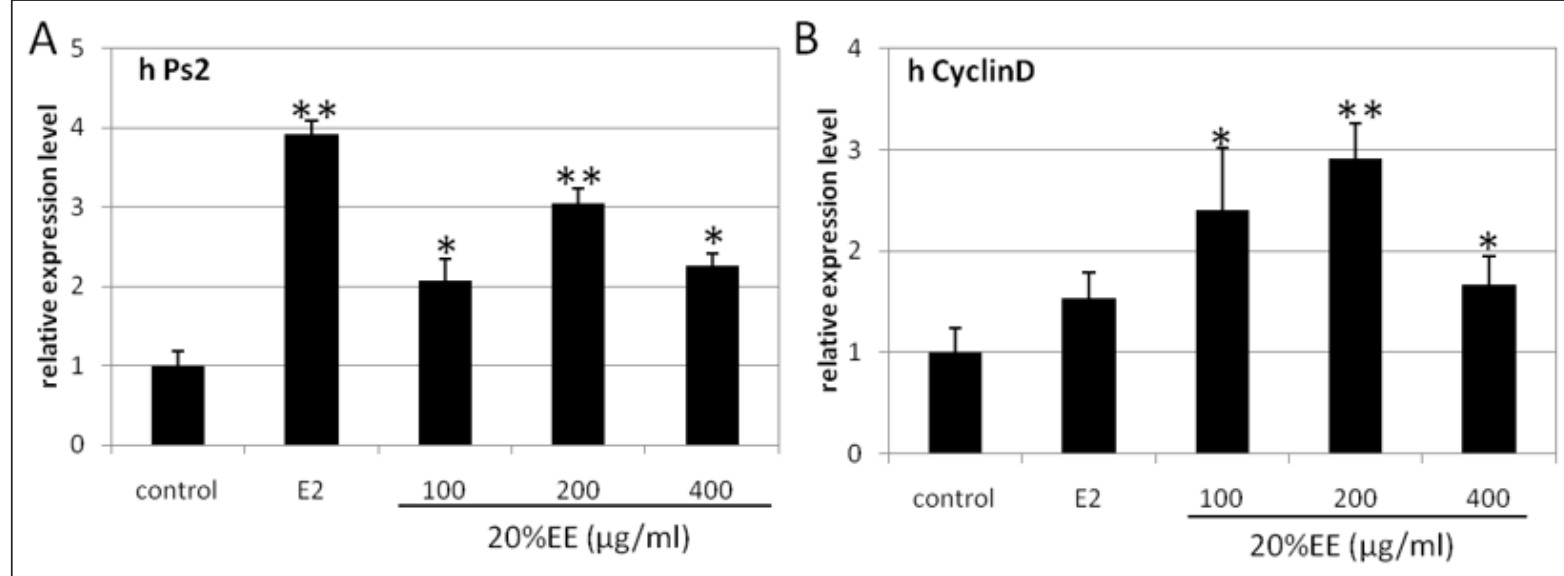

Figure 5: Effects of the 20\% EE on the mRNA expressions of pS2 (A) and cyclin D (B) in MCF-7 cells. The signals were normalized to 18s internal control, and the results were expressed as fold induction in comparison to control (average value \pm SD). Values marked by *; **, are significantly different $(\mathrm{p}<0.05 ; \mathrm{p}<0.01)$.

\section{Conclusion}

In this study, the effects of solvent to sample ratio, temperature, extraction time, number of extractions and solvent type were studied for extraction of phenols and flavonoids from mycelia of Cordyceps Ophioglossoides. The total phenol and flavonoid content were 21.8 and $13.2 \mathrm{mg} / \mathrm{g}$ dry powder of mycelia. These results could be used on large scale extraction. We demonstrated that the aqueous and ethanol extracts of cultured Cordyceps Ophioglossoides present strong anti-oxidative in-vitro and estrogenic activities in human breast cancer cell lines for the first time. These activities suggest that the cultured mycelia of Cordyceps Ophioglossoides have a potential synergistic effect on women's health and should be considered as a potential medical resource.

\section{Author Contributions}

Yong-quan $\mathrm{Li}$ and Qinqin Xu contributed to conception and design of the study; Qinqin Xu, Zhao and Yisheng Sun contributed to acquisition of data; Qinqin Xu and Ryan P Mackay organized and wrote the manuscript; all the authors contributed to interpretation of the data and provided input for manuscript.

\section{Acknowledgment}

We are thankful to China Scholarship Council for financial support of this study. This work was also supported by the Research Project of Science and Technology of Zhejiang Province, China (No. 2005C23027), Administration of Traditional Chinese Medicine of Zhejiang Province (2014ZB093), China, NSFC projects (31370064) and the Science and Technology planning Project of Zhejiang Province, China (2017C33137).

\section{References}

1. Zang M, Xu XR, Ji DQ (1975) Yunnan economic fungus data. Acta Botanica Yunnanica 1: 28-34.

2. Liu B (1984) Medical fungi of China ( $3^{\text {rd }}$ edn). China: People's Publisher of ShanXi, China.

3. Xu Q Liu Z, Sun Y, Ding Z, Lu L, et all. (2012) Optimization for Production of Intracellular Polysaccharide from Cordyceps ophioglossoides L2 in Submerged Culture and Its Antioxidant Activities in vitro. Chinese Journal of Chemical Engineering 20(2): 294-301.

4. Xu Q Lu L, Chen S, Zheng J, Zheng G, et al. (2009) Isolation of Cordyceps ophioglossoides L2 from Fruit Body and Optimization of Fermentation Conditions for Its Mycelial Growth. Chinese Journal of Chemical Engineering 17(2): 278-285.

5. Huang S, Lin C, Mau J, Li Y, Tsai S (2015) Effect of UV-B Irradiation on Physiologically Active Substance Content and Antioxidant Properties of the Medicinal Caterpillar Fungus Cordyceps militaris (Ascomycetes). International Journal of Medicinal Mushrooms 17(3): 241-253.

6. Dong C, Yang T, Lian T (2014) A Comparative Study of the Antimicrobial, Antioxidant, and Cytotoxic Activities of Methanol Extracts from Fruit Bodies and Fermented Mycelia of Caterpillar Medicinal Mushroom Cordyceps militaris (Ascomycetes). International Journal of Medicinal Mushrooms 16(5): 485-495.

7. Dong C, Yao Y (2008) In vitro evaluation of antioxidant activities of aqueous extracts from natural and cultured mycelia of Cordyceps sinensis. Lwt-Food Science and Technology 41(4): 669-677.

8. Vinson J, Hao Y, Su X, Zubik L (1998) Phenol antioxidant quantity and quality in foods: Vegetables. Journal of Agricultural and Food Chemistry 46(9): 3630-3634.

9. Enayat S, Banerjee S (2009) Comparative antioxidant activity of extracts from leaves, bark and catkins of Salix aegyptiaca sp. Food Chemistry 116(1): 23-28. 
10. Juan MY, Chou C (2010) Enhancement of antioxidant activity, total phenolic and flavonoid content of black soybeans by solid state fermentation with Bacillus subtilis BCRC 14715. Food Microbiology 27(5): 586-591.

11. Fitzpatrick LA (1999) Selective estrogen receptor modulators and phytoestrogens: new therapies for the postmenopausal women. Mayo Clin Proc 74(6): 601-607.

12. Dixon RA (2004) Phytoestrogens. Annual Review of Plant Biology 55 225-261.

13. Daley J (2009) Phytoestrogens and hormonal health. Journal of Complementary Medicine 8(6): 30-33.

14. Brzezinski A, Debi A (1999) Phytoestrogens: the "natural" selective estrogen receptor modulators? Eur J Obstet Gynecol Reprod Biol 85(1): 47-51.

15. Brandi M (1999) Phytoestrogens and menopause. Environmental Toxicology and Pharmacology 7(3): 213-216.

16. Mau J, Chang C, Huang S, Chen C (2004) Antioxidant properties of methanolic extracts from Grifola frondosa, Morchella esculenta and Termitomyces albuminosus mycelia. Food Chemistry 87(1): 111-118.

17. Jia Z, Tang M, Wu J (1999) The determination of flavonoid contents in mulberry and their scavenging effects on superoxide radicals. Food

ISSN: 2574-1241

DOI: 10.26717/BJSTR.2018.11.002126

Yong Quan Li. Biomed J Sci \& Tech Res (C) This work is licensed under Creative

Submission Link: https://biomedres.us/submit-manuscript.php
Chemistry 64(4): 555-559.

18. Shimada K, Fujikawa K, Yahara K, Nakamura T (1992) Antioxidative properties of xanthan on the autoxidation of soybean oil in cyclodextrin emulsion. Journal of Agricultural and Food Chemistry 40 (6): 945-948.

19. Marklund S, Marklund G (1974) Involvement of the superoxide anion radical in the autoxidation of pyrogallol and a convenient assay for superoxide dismutase. Eur J Biochem 47(3): 469-474.

20. Smirnoff N, Cumbes QJ (1989) Hydroxyl radical scavenging activity of compatible solutes. Phytochemistry 28(4): 1057-1060.

21. Mosmann T (1983) Rapid colorimetric assay for cellular growth and survival: application to proliferation and cytotoxicity assays. J Immunol Methods 65(1-2): 55-63.

22. Al-Farsi M, Lee C (2008) Optimization of phenolics and dietary fibre extraction from date seeds. Food Chemistry 108(3): 977-985.

23. kim S, Han D, Moon K, Rhee J (1995) Measurement df Superoxide Dismutase-Like Activity of Natural Antioxidants. Bioscience Biotechnology and Biochemistry 59(5): 822-826.

24. Celik S, Ozyurek M, Guclu K, Apak R (2010) Solvent effects on the antioxidant capacity of lipophilic and hydrophilic antioxidants measured by CUPRAC, ABTS/persulphate and FRAP methods. Talanta 81(4-5): 1300-1309.

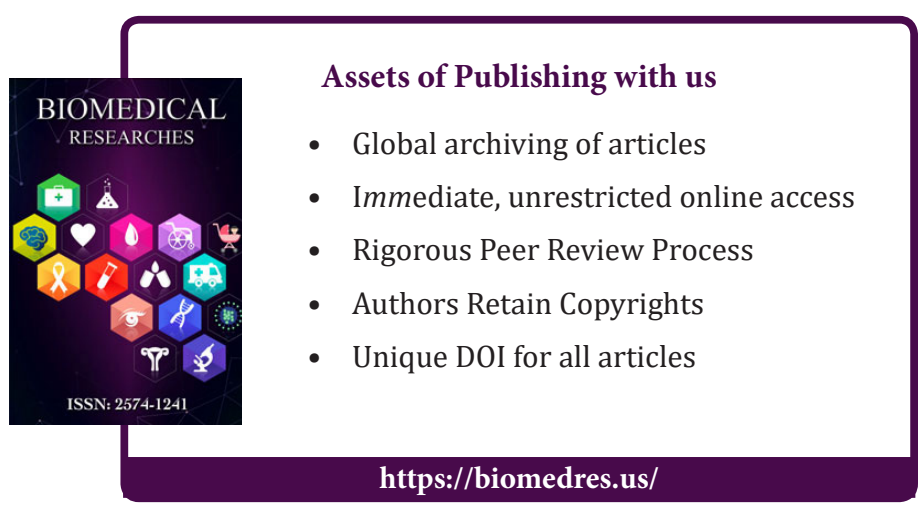

Cite this article: Qinqin Xu, Zhao Zhao, Yisheng Sun, Ryan P Mackay, Yong-Quan Li. Extraction Optimization for Phenols and Flavonoids from Cultured Mycelia of Cordyceps Ophioglossoides and Exploration of Bioactivities of its Aqueous and Ethanol Extracts. Biomed J Sci \& Tech Res 